\title{
Une ville vue par des étudiants en mobilité. Etude discursive de l'appropriation cognitive d'un nouvel espace urbain
}

\author{
Marge Käsper et Anu Treikelder (Université de Tartu, Estonie)
}

\begin{abstract}
The article explores how foreign students discover and experience the space of their hosting city, as reflected by their discourse about the common landmarks and places of the urban space. Our study concerns a specific social group that is in-between a local inhabitant and a tourist staying only a short time in a city - students in the situation of mobility. To study in what ways these students talk about the city, how they position themselves in respect of its space, adopting different viewpoints, we analyze, by a series of interviews conducted with them, how their discourse reveals the process of the appropriation of the hosting space. We examine first how the cognitive appropriation process of a city space is reflected in students' discourse in general, in what elements it appears and also how it is constructed and developed during the interview. We focus then on the answers to the question concerning a postcard representing Tartu where the discourse of students reveals the best the in-between status of the foreign students, standing between an exterior observer and an "expert" of the city. The most explicit fluctuation between the viewpoints is reflected in personal pronouns use, the more implicit ways are observed in the ways of describing the places, and in the argumentations about the discussed postcard. Furthermore, we also point out the impact of the interview as a disposal for interviewed persons to think about these spatial relations for themselves and for their perception of space in general.
\end{abstract}

Keywords: mobility students, urban space, identity discourse, personal pronouns, dialogue

\section{Introduction}

Quelle est la perception d'un espace connu pour nous par un étranger qui arrive dans ces lieux ? Comment se traduit plus particulièrement dans le discours l'expérience cognitive d'un espace urbain ? Dans une approche discursive, où « ce sont les discours qui font la ville » (Wald et Leimdorfer 2004, 265), nous allons présenter la conception et les premiers résultats des interviews menées avec des étudiants francophones à Tartu, en Estonie, pour étudier l'appropriation cognitive d'une ville par des étudiants étrangers qui y résident de manière temporaire.

Nous commençons par situer cette étude vis-à-vis d'autres travaux ayant fait croiser les problématiques d'espace urbain et d'identité dans le discours. Après avoir présenté ensuite notre corpus et mis l'accent sur le rapport énonciatif apparaissant dans le discours, nous présenterons comment, dans le cadre général de l'interview et dans les réponses à une 
question choisie, se manifestent et s'articulent ce que nous allons distinguer sommairement comme points de vue extérieur et intérieur dans l'appropriation cognitive d'une ville.

\section{2. Étudier le rapport entre l'espace et l'identité dans le discours}

\subsection{L'espace urbain et l'identité des étudiants en mobilité}

Dans l'espace urbain, l'intérêt des chercheurs a, ces derniers temps, souvent porté sur les banlieues et sur des quartiers plutôt difficiles des villes. Des approches par les mots en ont pu «nommer les divisions» (Topalov 2002), cerner des articulations sémantiques (BrancaRosoff 2001) ou montrer des variations langagières. Les effets de ségrégation et la sociolinguistique «de crise » restant d'actualité (Bulot et Bauvois 2002 ; Bulot et al. 2015), les études en sociolinguistique urbaine s'intéressent néanmoins également, et de plus en plus, à des réponses multiples et des hybridations tant langagières que culturelles qui se créent dans des flots migratoires de grandes villes. Dans la ville, se croisent les parcours, les expériences et les objets multiples (Smakman et Heinrich 2017), et les parcours particuliers, mis en mots de manière intime, peuvent contribuer à la compréhension "ancrée » des terrains d'étude (Vetier 2017). En définissant ci-dessous l'espace urbain par un petit groupe d'actants qui se situent par rapport à une ville où ils demeurent temporairement, nous nous intéresserons au rapport qu'ont ces actants à leur terrain d'action (dont font également partie, dans ce cadre, les actants sur ce terrain que sont les habitants réguliers de la ville en question).

Parmi les dialectiques de l'identité et de l'altérité qui peuvent «émerger » dans une ville (cf. Wald et Leimdorfer 2004), nous proposons donc de nous concentrer ici sur un « registre » particulier qu'est celui des étudiants étrangers dans une ville. "Entre la mobilité passagère du touriste et le déplacement à long terme du migrant » (Murphy-Lejeune 2003, 200), les étudiants en situation de mobilité constituent en effet un cas intéressant des flots migratoires d'une ville, notamment parce que « contrairement à l'immigré, l'étudiant sait qu'il rentrera chez lui [et] il jouit d'un statut globalement positif dans la société d'arrivée, qui lui ouvre un champ des possibles » (Papatsiba 2003, XIII).

Dans ces conditions, le rapport de cette «tribu» migratoire à son espace d'accueil ne se définirait pas tellement en termes d'affrontement, dans une situation conflictuelle, mais plutôt en termes de parcours, dans la mesure où c'est par des proximités sociales et spatiales que se crée un espace identitaire (cf. Wald et Leimdorfer 2004). Fred Dervin, qui a étudié le statut particulier des étudiants Erasmus à partir de témoignages d'anciens étudiants Erasmus laissés sur le site officiel du programme célébrant ses 20 ans en 2007 (Dervin 2007), et plus particulièrement, à partir d'un corpus d'entretiens avec des étudiants Erasmus français en Finlande (Dervin 2008), a confirmé en effet qu'en général, les étudiants sont très positifs visà-vis de leur séjour. Mais il a cependant noté qu'ils témoignent également d'importants malentendus sur la culture et l'identité (cf. également Buchart 2017). Avec Paganini-Rainaud (1998) et Papatsiba (2003), Dervin $(2007,66)$ constate que ces expériences se caractérisent souvent comme un discours dialectique où, d'une part, on peut parler de l'acceptation de la différence et d'un refus des stéréotypes, alors que d'autre part, ces discours sont "marqués par des commentaires amplement stéréotypés ». La question d'affrontement s'y retrouve donc, mais il faudra voir dans quelle mesure les malentendus et les stéréotypies rentrent dans les expériences reliées à l'espace urbain. 
Dans l'analyse des témoignages des étudiants Erasmus, Dervin met en avant la dimension de la découverte de soi, au détriment de celle des altérités. Pour notre étude, un tel constat suggère au terme de parcours plutôt un sens personnalisé - la ville d'accueil se définit pour l'étudiant avant tout par rapport à ses expériences antérieures, en tant qu'étape dans son parcours individuel d'études et de vie. Du point de vue des études urbaines, il est toutefois intéressant de savoir comment une ville d'accueil se définit sous cet angle de découverte. Comment les étudiants s'approprient-ils ce «champ des possibles »? Restent-ils plutôt des observateurs extérieurs par rapport à la ville donnée, pour ne véhiculer que des stéréotypes, ou bien adoptent-ils un point de vue interne, d'un connaisseur, qui parle de la ville comme de la sienne, appropriée par son expérience personnelle ? Vu le statut intermédiaire d'un étudiant étranger entre un touriste et un habitant local, nous supposons de fait qu'il s'énonce tantôt comme observateur extérieur qui vient découvrir cette ville, tantôt comme connaisseur ou expert qui s'est déjà approprié cet espace à sa manière et le présente comme le sien. Dans cette dimension spatiale de l'appropriation de l'espace donné, il est donc toujours intéressant de garder aussi la dimension spatiale du terme de parcours. Pour étudier ces parcours au sens multiple de manière bien située, nous allons nous concentrer sur les témoignages à propos d'une ville concrète, vue par un groupe d'étudiants choisi.

\subsection{Le corpus : les interviews pour décrire l'espace urbain de Tartu}

Déjà l'urbaniste Kevin Lynch, pour arriver à une Good City Form (Lynch 1981), en cherchant « les formes de base » de ce qui constitue une ville, s'est intéressé au « contenu que l'on peut rapporter aux formes physiques » (Lynch [1960] 1976, 54). Tout en partant des formes physiques d'une ville, pour cerner les manières de la catégoriser, il a mené des interviews avec ses habitants. Telle a été également la démarche dans un projet centré sur la ville de Montpellier, où une série d'interviews semi-directives a été menée entre autres dans l'ancien quartier central de cette ville pour étudier les conceptualisations et les rapports de ses habitants à celui-ci (cf. Barbéris 2005).

En automne 2015, huit étudiants francophones ${ }^{1}$ en mobilité à Tartu, en Estonie, ont participé à une enquête menée par Rebane (2016) sous forme d'interviews semi-directives : avec le micro ouvert, les entretiens de durée variable, en moyenne de 20 à 30 minutes, se sont déroulés sur la base de questions préparées mais selon un protocole libre quant à l'ordre et la forme de leur présentation. Plutôt que d'assembler des données précises, l'enjeu des interviews était en effet d'd'encourager les interviewés à s'exprimer de manière spontanée et de mettre ainsi en relief des variations dans les expériences.

Le nombre limité d'interviews est alors dû d'abord au fait qu'il s'agissait d'un projet à dominante qualitative et testant les moyens d'aborder la thématique. Toutefois, compte tenu du petit nombre de Français choisissant cette destination, l'échantillon est relativement représentatif. Avec ses 93000 habitants, Tartu n'est certainement pas une métropole, mais elle est la deuxième ville d'Estonie par sa taille, et elle est surtout très universitaire. Il y a plus de 10000 étudiants, ce qui représente un dixième de sa population. Les étudiants étrangers

\footnotetext{
${ }^{1}$ L'un des étudiants interviewés était grec, habitait à Athènes, mais il parlait français depuis l'âge de 5 ans. Les autres étaient français.
} 
(toutes provenances confondues) constituent à leur tour un dixième $\mathrm{e}^{2}$ de la population estudiantine.

En ce qui concerne les villes de provenance des étudiants interviewés, ils venaient pour la plupart de grandes villes comme Paris, Lyon, Toulouse, Dijon, ou encore Athènes, par rapport auxquelles Tartu est certainement une petite ville. Les deux villes de provenance les plus petites étaient Niort et Angers (les deux d'une population d'environ 150000 habitants). Quant aux parcours personnels d'études de ces étudiants, deux d'entre eux préparent une formation à l'enseignement, deux étudient la technologie de l'information ou le droit, un l'économie ou les relations internationales. Parmi les enquêtés, il y a cinq femmes et trois hommes, deux d'entre eux connaissaient l'Estonie depuis deux ans, les autres depuis quelques mois.

Les parcours de mobilité concernés sont donc assez variés, et nous ne pourrons pas mettre en relief toutes ces variables ici, mais nous pouvons affirmer d'ores et déjà que l'expérience de l'interview qualitative montre que la perception et le rapport à l'espace d'accueil varie le plus en fonction des personnalités concernées - les intérêts variaient des boites de nuit aux promenades dans la nature (pour plus de détails, Rebane 2016). Le point commun chez les interviewés était tout de même qu'en général ils se plaisaient bien à Tartu, qu'ils trouvaient « petite, mais très dynamique ».

Les questions portaient notamment sur les émotions et les avis des étudiants à l'arrivée et sur ceux de quelques mois plus tard, sur les routines qui s'étaient installées chez eux (cf. Barbéris 2005), sur leurs endroits préférés et sur leur appréhension de quelques endroits concrets. Nous n'en décrirons que quelques-unes, pour nous concentrer ensuite plus particulièrement sur une question - sur la représentation symbolique de Tartu au moyen d'une carte postale -, parce que ce sont les réponses à cette question qui témoignent des variations et des discussions les plus significatives pour ce qui concerne le rapport des énonciateurs à la ville d'accueil en question.

\subsection{Les points de vue dans l'énonciation}

Ce corpus serait une source intéressante pour différents types de recherche, dont par exemple les caractéristiques interactionnelles ou les éventuels effets de politesse apparaissant dans les interviews. Par exemple, l'enquêtrice étant un représentant de la communauté d'accueil, certaines contraintes de politesse sont susceptibles de surgir dans les réponses quant aux représentations évoquées et un positionnement vous $<>$ nous peut facilement entrainer des stéréotypes. Barbéris (2005) souligne en effet que dans l'interaction verbale entre enquêteur et enquêté, s'emboitent les dimensions dialogale et dialogique: alors que la succession des répliques fait émerger une dynamique dialogale, à l'intérieur du discours développé dans chaque réplique, chaque fois que celui-ci intègre des discours autres, une dynamique dialogique (au sens bakhtinien du terme, cf. Barbéris 2005, 39) fait émerger des représentations d'avant et d'ailleurs.

Du point de vue dialogal, il convient d'admettre ici que notre enquêtrice intervient assez peu après avoir posé la question. Dans l'interprétation des réponses, il faut néanmoins se rappeler que compte tenu du protocole libre de l'interview, pour favoriser l'interaction, les

\footnotetext{
${ }^{2}$ Selon les statistiques disponibles sur le site de l'Université de Tartu, il y avait en 2016 au total 12 970 étudiants, dont 980 étudiants étrangers ; en 2017 au total 9882 étudiants, dont 739 étudiants étrangers.
} 
questions ne sont pas posées dans le même ordre à tous les enquêtés et la forme des questions varie un peu. Quant aux positionnements respectifs des interlocuteurs, on peut dire que même si l'enquêtrice (une étudiante estonienne) et les enquêtés (étudiants français) représentent des communautés culturelles et linguistiques différentes, ils ont le même statut social à titre d'étudiants et se sentent ainsi plutôt à l'aise dans leur expression (Les interviewés peuvent se sentir peut-être même en situation de supériorité du point de vue linguistique.)

Du point de vue dialogique, le discours des interviewés est donc susceptible de refléter divers positionnements. Nous n'allons toutefois pas forcément chercher à cerner ces hétérogénéités énonciatives. Dans cette étude, nous nous focalisons sur les aspects liés à la prise de position des étudiants enquêtés en tant qu'énonciateurs par rapport à leur objet du discours (l'espace d'accueil).

$\mathrm{Vu}$ leur rapport particulier à l'espace d'accueil (cf. 2.1), nous avons avancé l'hypothèse que le discours des étudiants en mobilité révèle leur statut intermédiaire, les faisant apparaître tantôt comme étrangers et observateurs extérieurs, porteurs d'un point de vue pour ainsi dire externe, tantôt comme faisant partie de la communauté locale, représentant un point de vue interne. Nous allons donc étudier, en particulier, de quelle manière cette prise de position se manifeste dans les propos des enquêtés. Le terme général de point de vue est ainsi utilisé ici de manière assez similaire à celle des études énonciatives textuelles : il renvoie à «une source énonciative particulière et indique, explicitement ou implicitement, ses représentations, et, éventuellement, ses jugements sur les référents » (cf. Rabatel 2007, 353). Par contre, les qualifiants externe et interne ne correspondent pas exactement à leur emploi dans ces approches. Ils ne servent pas à distinguer les différentes « voix » dans les propos des interviewés, que nous ne cherchons pas à identifier dans cette étude, ni la vision « objectivante » ou « subjectivante » de l'objet (du discours) (cf. Rabatel 2007, 356). Nous les employons ici pour désigner les propriétés d'une seule source énonciative qui se place à l'extérieur ou à l'intérieur de l'objet du discours (la ville de Tartu) et nous en cherchons les indices dans les réponses des interviewés. Au cours de l'interview, l'enquêté s'énonce comme un je qui peut (mais ne doit pas) s'opposer à vous désignant les habitants de Tartu ou les Estoniens en général incluant l'enquêtrice, mais peut également arriver à un nous incluant l'enquêtrice et les autres habitants de Tartu. Nous estimons que les deux cas, quel que soit le point de vue (externe ou interne) adopté, témoignent de l'appropriation (ou du rejet) de l'espace d'accueil par les étudiants: il s'agit simplement de deux manières différentes d'appréhender un objet du discours.

\section{Analyses}

\subsection{Du point de vue externe vers un point de vue interne?}

Nous avons déjà constaté ci-dessus que les circonstances interactionnelles favorisent clairement un point de vue externe des enquêtés. Les enquêtés avaient été prévenus qu'ils seraient questionnés sur leur ville d'accueil et ils savent que l'enquêteur représente la communauté locale, ils se positionnent donc a priori comme «étrangers ». D'autre part, l'apparition des points de vue est fortement tributaire du type de questions posées. Il n'est donc pas étonnant de voir surtout le point de vue externe dominer au début des interviews où sont posées les questions : De quelle ville tu viens? Quelles étaient tes premières impressions à ton arrivée à Tartu? Est-ce qu'il y avait quelque chose d'étonnant pour toi ? Dans les réponses à ces questions, on trouve beaucoup d'indices explicites d'un point de vue externe, 
surtout dans les comparaisons de Tartu à l'espace de provenance des étudiants, comme dans l'exemple (1) :

(1) $[\ldots]$ mm qu'il n'y a pas (.) en France souvent quand on est dans un village ou dans une ville sur la place principale il y a souvent les églises dans le centre vraiment (.) de la ville et ici à Tartu les églises (.) c'est pas un pays (.) Estonie n'est pas un pays très religieux donc les églises sont un peu excentrées (.) c'est aussi un peu étonnant pour nous (.) l'architecture qui est quand même assez différente parce qu'on est habitué plutôt à des bâtiments en pierre

Dans l'exemple (1), on trouve un «nous » (étonnant pour nous) ainsi qu'un « on » (on est habitué) référant directement à l'espace d'origine de l'enquêté. Le point de vue externe s'exprime explicitement par l'emploi des formes du pronom personnel et par les oppositions exprimées par les compléments de lieu (en France, ici à Tartu). Ce type de comparaisons est le plus souvent très général (en Estonie $v s$. en France) et ne concerne pas les villes/endroits précis.

Un autre type d'exemple de point de vue externe explicite vient des témoignages des changements que les étudiants ont dû subir pour s'adapter à leur espace d'accueil et qui impliquent une comparaison avec l'espace de provenance. Ces changements peuvent concerner leur comportement sous l'influence des habitants locaux, comme dans (2) où l'enquêté s'est mis à respecter le feu rouge, parce que « les Estoniens me regardaient »:

(2) $[\ldots]$ cela m'a changé du coup je respecte le feu rouge maintenant

D'autre part, les changements se manifestent aussi à un niveau plus général de la perception de l'espace, comme dans les exemples (3) et (4) où les enquêtés expriment leur adaptation aux dimensions de la ville, en répondant à la question Pour toi, Tartu est-ce une petite ville ou bien un peu grande aussi ? :

(3) [...] j'ai dû m'adapter [à calculer autrement la distance, puisque les numéros de maisons avancent lentement]

(4) je trouve que c'est grand au final parce que je me suis adapté avec les proportions les proportions que j'avais avant ont changé (.) et du coup (.) quand je vais par exemple à Põlva ou à Elva je me dis que oui ce sont des petites villes par rapport à Tartu (.) au final c'est la deuxième plus grande ville d'Estonie, comme Lyon est la deuxième plus grande ville de France (.) essentiellement ils sont presque similaires.

Les points de vue changent néanmoins petit à petit au cours de l'interview. Au fur et à mesure, les interviewés se construisent leurs repères spatiaux, et se positionnent par rapport aux lieux dont ils prennent conscience en tant qu'observateurs internes.

\subsection{Les points de vue sur une carte postale}

Pour les changements de points de vue, il est intéressant d'étudier surtout la question : Comment tu décrirais cette carte postale? Est-ce que tu penses que c'est une bonne représentation de la ville de Tartu? Quels objets ou lieux tu mettrais sur une carte postale de Tartu? 
L'enquêteur a montré aux étudiants une carte postale représentant trois endroits de Tartu et leur a posé ces questions assemblées, de manières légèrement différentes dans les interviews. Il s'agit donc en fait de 3 sous-questions :

- description /identification des lieux représentés ;

- évaluation de la carte en question ;

- propositions personnelles pour faire une carte postale de Tartu.

En fonction de la forme des questions, les réponses varient aussi un peu. La première et la dernière sont des questions ouvertes, donc potentiellement menaçantes pour la face de l'enquêté, le mettant dans l'impossibilité de ne pas développer sa réponse (cf. Maingueneau 2005 : 23-24). Il n'est pas possible de répondre par un simple «non », comme c'est le cas par exemple, à la question : Est-ce qu'il y a un lieu qui te fait peur/ qui ne te plaît pas?.

Dans le déroulement dialogal, cette question vient généralement au milieu de l'interview (l'ordre des questions varie), les enquêtés se sentent probablement plus à l'aise qu'au tout début; plusieurs endroits de Tartu ont déjà été évoqués dans les questions précédentes (y compris la place centrale qui figure sur la carte postale) et cela est susceptible d'influencer un peu les réponses.

Quant aux positionnements, c'est une question qui invite les enquêtés par sa forme même à s'énoncer comme connaisseur ( $v s$. observateur externe) ; a priori, on pourrait donc s'attendre à ce que les réponses reflètent au moins dans une certaine mesure un point de vue interne adopté par les enquêtés. D'autre part, ils doivent se mettre dans la position d'un Autre qui, dans cette question, n'est pas le représentant de la communauté locale (de Tartu ou plus généralement estonienne) dont l'enquêteur fait partie, mais quelqu'un qui ne connaît pas Tartu et qui devrait acquérir une bonne représentation de cette ville en regardant la photo proposée. C'est dans cette perspective que se réintroduit dans les réponses un point de vue externe, mais cette fois-ci externe aussi bien à l'interviewé qu'à l'intervieweur - c'est celui d'un Autre qui doit se représenter ou s'imaginer Tartu. On trouve ainsi des indices du point de vue externe, exprimé par le vous dans l'exemple (5), où dans les faits, l'enquêté adopte une position de connaisseur et propose des choses à représenter sur une carte postale :

(5) $[\ldots]$ peut-être la nourriture (.) peut-être une table estonienne (.) qu'est-ce qu'on a habitude de manger (.) même si vous n'êtes pas réputés pour votre gastronomie parce que c'est un mélange et il n'y a pas de gastronomie spécifique vraiment

Les exemples de regard externe des étudiants Erasmus eux-mêmes ne manquent toutefois pas non plus. Et, il y a un cas qui peut même être qualifié comme refus d'assumer le rôle de connaisseur :

(6) [...] c'est vrai que (.) à Tartu y a pas grand-chose pour représenter Tartu parce que c'est une petite ville et au final il faut connaître la ville pour la représenter...

Néanmoins, certains indices confirment de manière assez sûre l'apparition du point de vue interne.

On peut déjà trouver quelques détails « connaisseurs » dans les descriptions de la carte postale, comme dans (7) et (8) :

(7) c'est au printemps j'ai l'impression il y a du soleil donc ça ... 


\section{(8) selon moi c'est pris en été parce que les couleurs sont assez vives}

Ces propos indiquent que les énonciateurs ne sont pas seulement de passage, mais qu'ils ont vu l'alternance de saisons à Tartu. Il est vrai cependant que ces descriptions (il y a $d u$ soleil, les couleurs sont vives) peuvent aussi bien être déduites des indices extérieurs. Aucun enquêté (ni d'ailleurs l'enquêteur) ne reconnaît la gargouille présentée sur l'une des trois images sur la photo et certains enquêtés sont assez gênés par ce fait (l'enquêteur éprouve la nécessité de le rassurer en disant qu'elle ne la reconnaît pas non plus).

Mais c'est surtout le regard critique que les enquêtés adoptent en évaluant la carte postale qui les met en position de connaisseur et provoque un positionnement à l'intérieur de l'espace d'accueil. En effet, c'est l'un des rares cas où les critiques apparaissent au cours des interviews. En général, dans les autres questions, il s'agit de « descriptions en euphorie » dans les termes de Barbéris (2005) : on trouve rarement des remarques négatives. Généralement, les enquêtés ont du mal à trouver des réponses même aux questions qui concernent directement les aspects négatifs (de type : est-ce qu'il y a quelque chose qui ne te plaît pas ?) ; il y a beaucoup d'hésitations dans ces réponses. Quelques aspects négatifs surgissent surtout par rapport aux relations avec les habitants locaux (les caissières qui ne sourient pas, la difficulté de se faire des amis, etc.). Tandis que pour cette question, il y a plusieurs réponses critiques par rapport à la carte postale évaluée. Même si des moyens d'atténuation (la forme conditionnelle, je pense que, un peu) sont utilisés, les évaluations sont néanmoins assez directes, comme dans l'exemple (9) :

(9) $[\ldots]$ je pense que c'est un peu simpliste

ou même formulées de manière catégorique sur un ton assuré :

(10) $[\ldots]$ pour moi ça ne représente pas exactement Raekoja plats [la place de l'hôtel de ville]

(11) [...] mais oui c'est pas représentatif de Tartu en fait parce que la photo de Raekoja plats devrait être différente

Il est vraisemblable que les critiques sont plus nombreuses parce que les enquêtés ne menacent pas directement la face de l'enquêteur. C'est l'auteur " anonyme » de la carte postale qui est critiqué. Un enquêté désigne cette instance par «ils» (ils oublient ...ils ne montrent pas...); les autres la laissent plutôt impersonnelle (par exemple la photo est prise...).

Les critiques peuvent concerner la disposition des objets ou lieux sur la carte (cf. les exemples 10 et 11 ci-dessus), mais également le choix des objets et porter donc aussi sur la valeur esthétique ou représentative de ces objets, comme dans les exemples (12) et (13) :

(12) je dirais que c'est pas forcément les les plus jolis endroits à Tartu qui sont mis en valeur (l'évaluation porte sur le pont représenté sur la carte postale)

(13) et ce pont ben il est pas très joli il est en béton

Les critiques peuvent concerner également la ville en entier :

(14) c'est pas une ville extraordinaire sur le plan architectural ou sur le plan (.) voilà c'est pas effectivement forcément très très belle 
Quoique le même enquêté atténue tout de suite son évaluation :

(15) mais en revanche on a plein d'émotions ce qui a beaucoup de valeur

Par ailleurs, le statut «connaisseur» des enquêtés devient aussi manifeste dans les réponses à la troisième sous-question: qu'est-ce que tu mettrais sur une carte postale représentant Tartu?

Il est à noter que les propositions des enquêtés sont en forte corrélation avec les réponses à une question précédente qui concernait leur endroit préféré à Tartu. Dans la suite de l'interview, presque tous les enquêtés mentionnent alors cet endroit aussi parmi les lieux ou objets à mettre sur la carte postale.

Ce qui est le plus intéressant ici, c'est la manière dont les interviewés justifient leurs choix. Il y a deux types de justifications : les justifications qui peuvent être considérées comme générales, se basant sur un raisonnement (pourquoi ces lieux sont importants du point de vue culturel ou autre) ; et les justifications personnalisées qui évoquent explicitement les liens personnels des enquêtés avec les lieux mentionnés. On dirait cependant que dans les deux cas, les interviewés s'énoncent en connaisseurs et témoignent de l'appropriation de l'espace d'accueil.

Parmi les justifications personnalisées, certaines se basent sur les émotions que les enquêtés éprouvent envers les endroits proposés :

(16) je mettrais la cathédrale parce que justement je l'aime beaucoup

(17) la rivière c'est agréable

(18) j'aime beaucoup cette rivière ... ; et puis il manque ma fontaine non ça c'est vrai

Dans l'exemple (18), l'enquêté fait référence à sa réponse à la question sur son endroit préféré, en utilisant le déterminant possessif $m a$. Du point de vue dialogal, le possessif ne sert que d'anaphore rappelant l'objet déjà évoqué, dans la dimension dialogique, une telle désignation rejoint par contre les discours hypocoristiques reliés aux jouets favoris cognitivement bien appropriés ou autres objets de discours faisant émerger des émotions agréables (cf. Fèvre-Pernet 2004).

Les justifications personnalisées peuvent également s'inspirer des pratiques personnelles de l'enquêté (exemples 19 et 20) ou des pratiques du groupe (exemple 21) :

(19) c'est là où je viens me promener tous les jours (.) c'est là où je passe tous les jours (il s'agit de la place de l'hôtel de ville dans cet exemple)

(20) la fontaine aussi parce que je passe tous les jours devant

(21) pour moi peut-être aussi l'université étant donné que c'est là qu'on passe la plupart de notre temps

Dans l'exemple (21), il convient aussi de relever l'emploi du pronom on et du possessif notre (temps) qui ne renvoient certainement pas à l'espace de provenance de l'enquêté (comme c'était le cas dans l'exemple 1 présenté plus haut), mais à une instance vague qui peut référer à un groupe plus ou moins grand (aux étudiants Erasmus, aux étudiants de l'Université de Tartu incluant l'enquêteur, aux habitants de Tartu en général). Dans tous 
les cas, ces marques révèlent que l'enquêté se considère comme faisant partie d'une communauté locale.

Les justifications générales données peuvent résulter de différents types de raisonnements. Dans l'exemple (22), on voit un raisonnement que l'on pourrait appeler méronymique (Barbéris 2005 ; Branca-Rosoff 2001), relevant les lieux/objets comme une partie de l'espace de la ville (ou de l'espace de l'Estonie en général) :

(22) ce qui manque le plus c'est l'université parce que c'est super important et surtout il manque les étudiants je trouve que quand je vois cette université [xxx] elle est vraiment belle c'est vraiment une partie importante de la ville (.) puis aussi quand même la meilleure université de l'Estonie donc ça a quand même un impact (.) c'est une partie de la culture estonienne.

D'autres raisonnements pourraient être considérés comme métonymiques, étant donné que c'est la valeur symbolique des objets/endroits qui est soulignée. Les exemples 23 et 24 indiquent tels éléments de Tartu :

(23) la fontaine aussi c'est vraiment ce qui est une chose typique ;

(24) je pense aussi qu'ils oublient de mettre les maisons typiques dans les quartiers qui ont beaucoup de couleurs

C'est leur typicalité qui les fait représenter «sous forme d'image à l'intérieur d'un groupement caractéristique » comme l'unité thématique de la ville Lynch ([1960] 1976, 79). Alors que selon Lynch (ibid.) l'unité thématique d'un quartier de Boston serait constituée « d'étroites rues escarpées, des alignements de vieilles maisons de briques à l'échelle humaine, [...] de la tranquillité et des passants appartenant à la haute Société. », dans l'image de Tartu pour les étudiants français interviewés, la fontaine centrale et les maisons colorées constituent ainsi des unités thématiques importantes.

\section{Conclusion}

L'espace urbain de Tartu se décrit et se figure pour les étudiants français en mobilité donc effectivement, comme l'avait indiqué Lynch (1960), par des points de repère pratiqués (la fontaine centrale) et par un contenu personnalisé (les émotions, les gens). L'appropriation cognitive de l'espace urbain se manifeste alors dans le discours de manière explicite, reflétant un point de vue adopté interne. Une appropriation implicite de celui qui connait se manifeste toutefois aussi dans des réflexions plutôt généralisantes, où on caractérise Tartu par des unités thématiques perçues ou même en parlant des pratiques humaines ou des traditions du point de vue énonciativement externe. Il se peut par ailleurs qu'avant l'interview, les enquêtés n'aient pas spécialement réfléchi à des endroits spécifiques de Tartu. Ils s'attendent sans doute à parler des stéréotypes (Mais qu'est-ce que tu penses des Estoniens en général est-ce que c'est plutôt facile ou difficile de faire des amis ? - ouais (.) j'étais sûr que cette question allait apparaître quelque part). Mais dans la mesure où la saillance en termes de typicalité renvoie à une réflexion sur cet espace et sur leur rapport à cet espace, on peut affirmer que les parcours personnalisés des étudiants dans leur espace d'accueil auront contribué, d'une part, à leur appréhension intime de cet espace et, d'autre part, aussi à l'évolution de leurs parcours personnels, pour relativiser les perceptions. Au final, un témoignage comme je me suis adapté avec les proportions les proportions que j'avais avant ont changé (l'exemple 4) marque une 
appropriation de l'espace vécu mais aussi de l'appropriation de soi-même. Enfin, on peut souligner de même le moyen d'interview comme dispositif de co-construction du rapport étudié - c'est en effet au cours de l'interview qu'évoluent aussi les points de vue reflétés.

\section{Bibliographie}

Barbéris, J.-M., 2005. La ville et ses composantes : l'émergence des catégories en interaction orale. Revue de l'Université de Moncton 36 : 31-60. DOI : 10.7202/011988ar.

Branca-Rosoff, S., 2001. La sémantique lexicale du mot "quartier" à l'épreuve du corpus Frantext (XII $-\mathrm{XX}^{\mathrm{e}}$ siècles). Langage et société 2001/2 (96): 45-70. DOI : 10.3917/1s.096.0045.

Buchart, M., 2017. Expérience de l'altérité et discours sur la rencontre interculturelle. Le cas des étudiants de FLE finlandais. Résumés en ligne du XXe congrès des romanistes scandinaves, du 15 au 18 août 2017, Université de Bergen, 99-100. http://www.uib.no/en/node/97866\#book-of-abstracts.

Bulot et al., 2015 = Bulot, T., I. Boyer, et M.-M. Bertucci (éds.) 2015. Villes, discours, ségrégation, minorités. Cahiers de Linguistique 41/1.

Bulot, Th., Bauvois, C., 2002. La sociolinguistique urbaine : une sociolinguistique de crise ? Premières considérations. Marges Linguistiques 3/2002 : 7-13.

Dervin, F., 2007. Erasmus : 20 ans d'hypermobilité/hypomobilité existentielle ? Synergies. Pays Riverains de la Baltique 4/2007 : 63-78.

Dervin, F., 2008. Métamorphoses identitaires en situation de mobilité. Thèse de doctorat. Paris III /Turun Yliopisto. http://www.theses.fr/2008PA030027.

Fèvre-Pernet, Ch., 2004. Procédés dialogiques dans la (dé)nomination d'objets manufacturés : les noms de jouets. In Cassanas et al. (2004) Dialogisme et nomination. Montpellier : Université Paul-Valéry, Montpellier III. 269-282.

Lynch, K., 1981. Good City Form. Cambridge MA and London: MIT Press.

Lynch, K., [1960] 1976. The Image of the City. [Trad. fr. 1976. L'image de la cité]. Paris : Dunod.

Maingueneau, D., [1998] 2005. Analyser les textes de communication. Paris : Armand Colin.

Murphy-Lejeune, E., 2003. L'étudiant européen voyageur, un nouvel étranger. Paris : Didier.

Paganini-Rainaud, G., 1998. Entre le «très proche» et le «pas assez loin » : différences, proximité et représentation de l'italien en France. Thèse de doctorat. Paris III.

Papatsiba, V., 2003. Des étudiants européens : “Erasmus” et l'aventure de l'altérité. Bern : Peter Lang.

Rabatel, A., 2007. Analyse énonciative du point de vue, narration et analyse de discours. Filologia e Lingüística Portuguesa : 345-368. <halshs-00367547>.

Rebane, E., 2016. Tartu vu par les étudiants Erasmus francophones. Analyse discursive de la perception de l'espace urbain. Mémoire de licence, Université de Tartu. http://hdl.handle.net/10062/51925. 
Smakman, D., Heinrich, P., 2018. Urban Sociolinguistics: The City as a Linguistic Process and Experience. New York : Routledge.

Topalov, Ch., 2002. Langage, société et divisions urbaines. Les divisions de la ville [en ligne]. Paris: Éditions de la Maison des sciences de l'homme. http://books.openedition.org/editionsmsh/1254.

Vetier, Th., 2017. Donner à voir l'intime dans l'espace public : réflexions épistémologiques et recherche appliquée en sociolinguistique urbaine. Sociolinguistique urbaine. A la mémoire de Thierry Bulot 1/2017 (3) : 152-162.

Wald, P., Leimdorfer, F. (éds.) 2004. Parler en ville, Parler de la ville, Essais sur les registres urbains. Paris : Éditions UNESCO / Éditions de la Maison des sciences de l'homme. 